\title{
Strangeness in high-energy nuclear collisions
}

Marco van Leeuwen* for the NA49 collaboration

NIKHEF, Amsterdam, Netherlands E-mail: 'mvi@onikhef

ABstract: Recent data from NA49 on strangeness production in nuclear collisions at the SPS are presented. Kaon rapidity spectra at $40 A \cdot \mathrm{GeV}$ are shown and the kaon to pion ratio is compared to other energies. There is an indication of a maximum in the $\left\langle K^{+}\right\rangle /\left\langle\pi^{+}\right\rangle$ratio near $40 \mathrm{~A} \cdot \mathrm{GeV}$. Measurements of the mid-rapidity $\Lambda$ and $\Xi$ yields in $\mathrm{p}+\mathrm{p}$ and $\mathrm{p}+\mathrm{A}$ collisions at $158 \mathrm{~A} \cdot \mathrm{GeV}$ show that the hyperon yields increase faster than the number of participants. The $\phi$-yield has been measured for $\mathrm{Pb}+\mathrm{Pb}$ collisions at different centralities and for central $\mathrm{C}+\mathrm{C}$ collisions at $158 \mathrm{~A} \cdot \mathrm{GeV}$. The yield in $\mathrm{C}+\mathrm{C}$ collisions is higher than in $\mathrm{Pb}+\mathrm{Pb}$ interactions with the same number of participants.

\section{Introduction}

The aim of research in the field of high-energy heavy ion collisions is to find and characterise the transition from confined hadronic matter to deconfined quark matter, the Quark Gluon Plasma (QGP). Calculations of QCD on the lattice indicate that the phase transition should

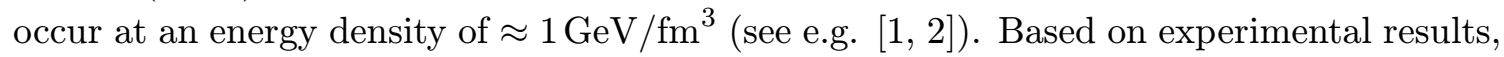
the energy density at the highest SPS energy $(158 \mathrm{~A} \cdot \mathrm{GeV})$ is estimated to be $3 \mathrm{GeV} / \mathrm{fm}^{3}$ [3i], well above the threshold energy. In order to search for signs of a transition, NA49 has recently taken data at lower energies $(40$ and $80 A \cdot G e V)$. In addition, different system sizes $(\mathrm{p}+\mathrm{p}, \mathrm{p}+\mathrm{A}, \mathrm{C}+\mathrm{C}, \mathrm{Si}+\mathrm{Si}$, and $\mathrm{Pb}+\mathrm{Pb}$ at various centralities) are studied at $158 \mathrm{~A} \cdot \mathrm{GeV}$.

A selection of the most recent results of NA49 on strangeness production as a function of beam energy and system size will be presented. An increase in strangeness production was originally proposed as a signature of the QGP, because the equilibration of strangeness in a QGP is much faster than in a hadron gas [4i-4] energy, where there is certainly no QGP formation, the relative strangeness content in nuclear collisions is already much higher than in elementary collisions. NA49 is studying kaon production at several different energies to see whether there is any indication of a transition when going to higher energies. The recent result at $40 \mathrm{~A} \cdot \mathrm{GeV}$ is a first measurement which bridges the gap between AGS and SPS energies.

\footnotetext{
${ }^{*}$ Speaker.
} 
Results on hyperon production in $\mathrm{p}+\mathrm{p}$ and $\mathrm{p}+\mathrm{A}$ collisions and $\phi$ production in $\mathrm{Pb}+\mathrm{Pb}$ collisions of different centralities and in central $\mathrm{C}+\mathrm{C}$ collisions are presented to study the system size dependence of strangeness production.

\section{The experiment}

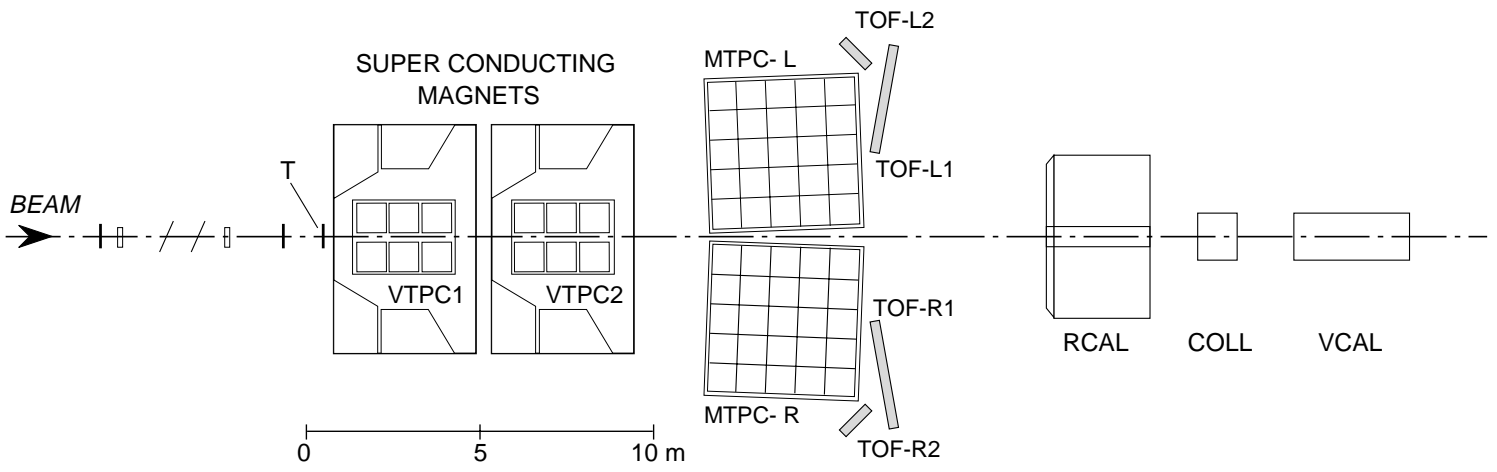

Figure 1: The NA49 experimental setup. Shown are the four TPCs (VTPC, MTPC), the dipole magnets and the different TOF systems. The veto calorimeter (VCAL) is used for the centrality determination in nuclear collisions.

The NA49 apparatus is a large acceptance hadronic spectrometer [inj] at the CERN SPS. The main detectors are four large TPCs, two of which are placed in a magnetic field (see Fig. (ii $)$. The specific energy loss $(d E / d x)$ is measured in the TPCs and used for particle identification. Two large time-of-flight (TOF) detectors provide additional particle identification around mid-rapidity. The detector system can be used for different beamtarget combinations. During $\mathrm{p}+\mathrm{Pb}$ running an additional detector is used to determine the centrality by measuring the knock-out of low-energy particles [īi] $]$.

\section{Results}

\subsection{Energy dependence of kaon production}

Kaons are an important measure of the total produced strangeness, since they carry most of the produced strangeness at AGS and higher energies. Kaons are identified in NA49 by $d E / d x$ and TOF. The rapidity spectra of $K^{+}$and $K^{-}$at $40 A \cdot \mathrm{GeV}$ are shown in Fig ${ }_{2 i}^{2}$ Note that the experimental acceptance covers most of the forward hemisphere. The energy dependence of the kaon to pion ratio in full phase space is shown in Fig $1 \overline{3}_{-}^{-}$The $\left\langle K^{-}\right\rangle /\left\langle\pi^{-}\right\rangle$ratio increases roughly monotonically with collision energy. The $\left\langle K^{+}\right\rangle /\left\langle\pi^{+}\right\rangle$ ratio in $40 \mathrm{~A} \cdot \mathrm{GeV} \mathrm{Pb}+\mathrm{Pb}$ collisions is the highest measured so far, although it is only slightly higher than at the highest AGS energy $(\approx 10 \mathrm{~A} \cdot \mathrm{GeV})$.

A peak in the $\left\langle K^{+}\right\rangle /\left\langle\pi^{+}\right\rangle$ratio between 10 and $40 A \cdot \mathrm{GeV}$ would imply that both the

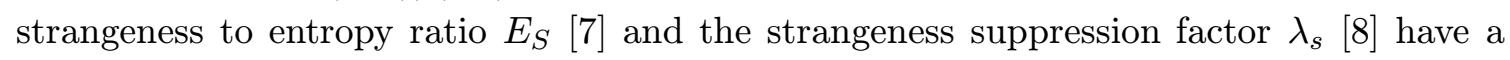
peak in this region, since the anti-strangeness in the final state is mostly carried by $K^{+}$ 


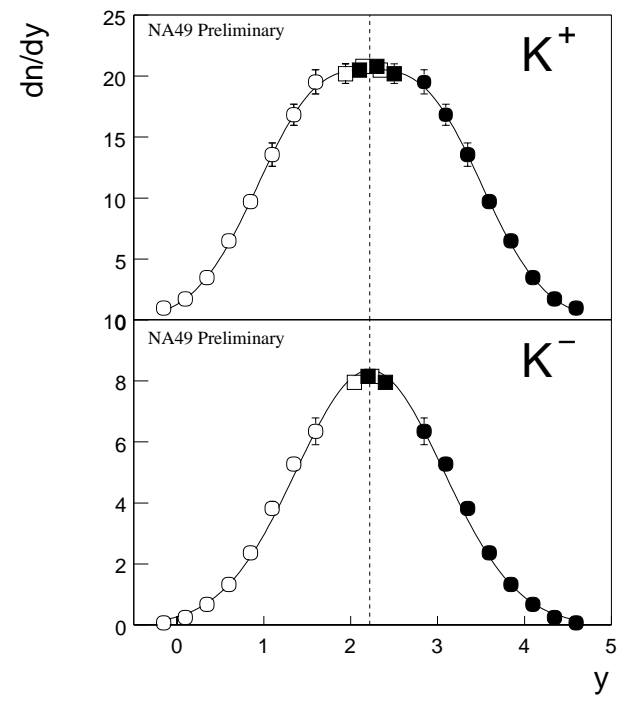

Figure 2: Rapidity distribution of kaons produced in central $\mathrm{Pb}+\mathrm{Pb}$ collisions at $40 \mathrm{~A}$. $\mathrm{GeV}$ beam energy. The closed points are the measured values, the open points are reflected around mid-rapidity.

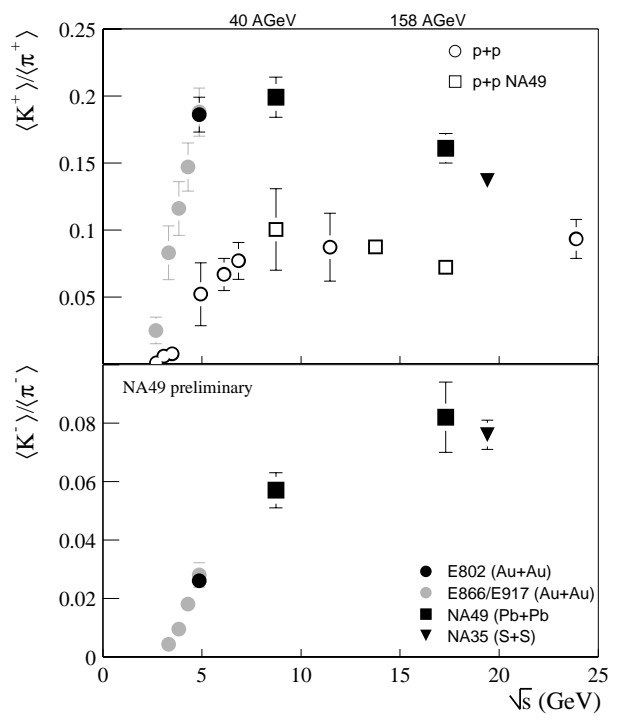

Figure 3: Energy dependence of kaon to pion ratios (full phase space) in central $\mathrm{Pb}+\mathrm{Pb}$ (and $\mathrm{Au}+\mathrm{Au}$ ) and minimum bias $\mathrm{p}+\mathrm{p}$ (open symbols) collisions for positive and negative particles separately.

(the anti-hyperon yields are very small compared to the $K^{+}$yield). In [i] it is argued that this could be an indication of the phase transition. First results on kaon production at $80 A \cdot \mathrm{GeV}$ will become available in the near future.

\subsection{Hyperon yields in $\mathrm{p}+\mathrm{p}, \mathrm{p}+\mathrm{A}$, and $\mathrm{A}+\mathrm{A}$ interactions}
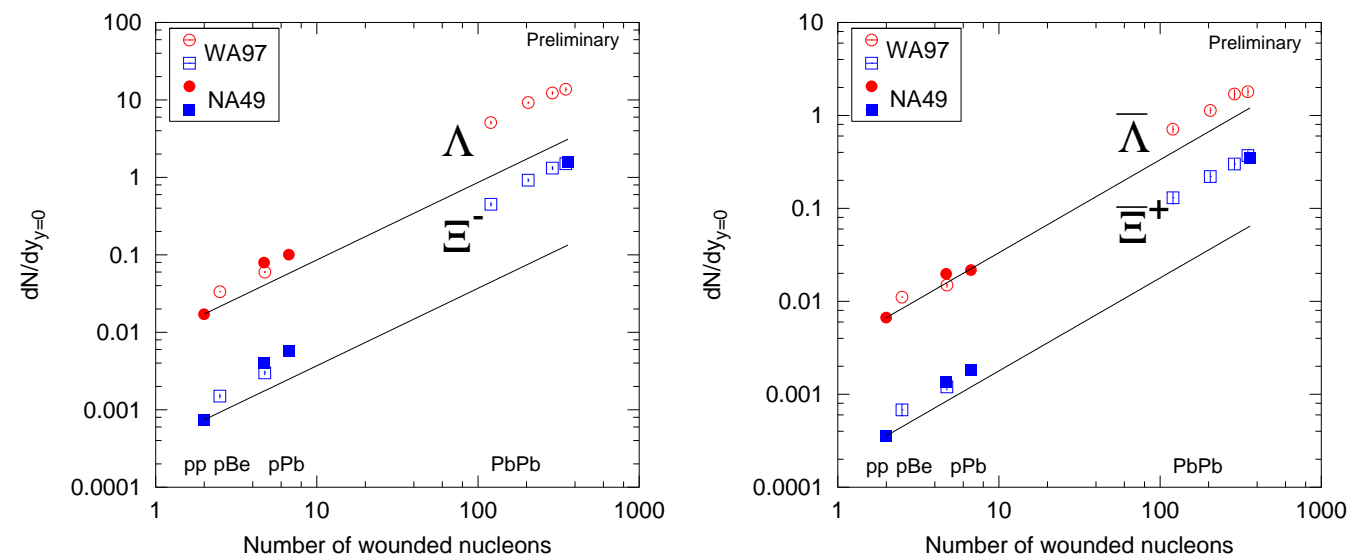

Figure 4: Mid-rapidity yields of (anti-)hyperons at $158 \mathrm{~A} \cdot \mathrm{GeV}$ beam energy as a function of system size. Results for $\mathrm{p}+\mathrm{p}, \mathrm{p}+\mathrm{Pb}$, and central $\mathrm{Pb}+\mathrm{Pb}$ from $\mathrm{NA} 49$ and for $\mathrm{p}+\mathrm{Be}, \mathrm{p}+\mathrm{Pb}$, and centrality dependent $\mathrm{Pb}+\mathrm{Pb}$ from WA97 are shown.

In Fig. in the $\Xi$ and $\Lambda$ yields at mid-rapidity in $158 \mathrm{~A} \cdot \mathrm{GeV} \mathrm{p}+\mathrm{p}$ and $\mathrm{p}+\mathrm{A}$ collisions are

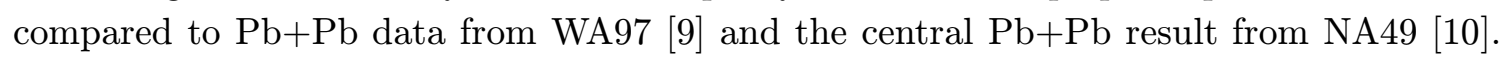


The lines show an extrapolation of the $\mathrm{p}+\mathrm{p}$ yields to $\mathrm{p}+\mathrm{A}$ and $\mathrm{A}+\mathrm{A}$, assuming that they are proportional to the number of participants. The enhancement of hyperon production per participant in $\mathrm{A}+\mathrm{A}$ collisions compared to $\mathrm{p}+\mathrm{A}$ collisions is clearly visible. There is, however also an enhancement when comparing $\mathrm{p}+\mathrm{p}$ and $\mathrm{p}+\mathrm{A}$ (except in the $\bar{\Lambda}$ which also has the smallest enhancement in $\mathrm{A}+\mathrm{A}$ ). One might wonder whether it is sensible to compare mid-rapidity yields of asymmetric $\mathrm{p}+\mathrm{A}$ collisions to (symmetric) $\mathrm{A}+\mathrm{A}$ or $\mathrm{p}+\mathrm{p}$ collisions. The effect of isospin on hyperon production is also not yet clear. Note that NA49 has measured hyperon yields in a larger interval around mid-rapidity (2-3 units), see

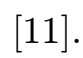

\subsection{System size dependence of $\phi$ production}
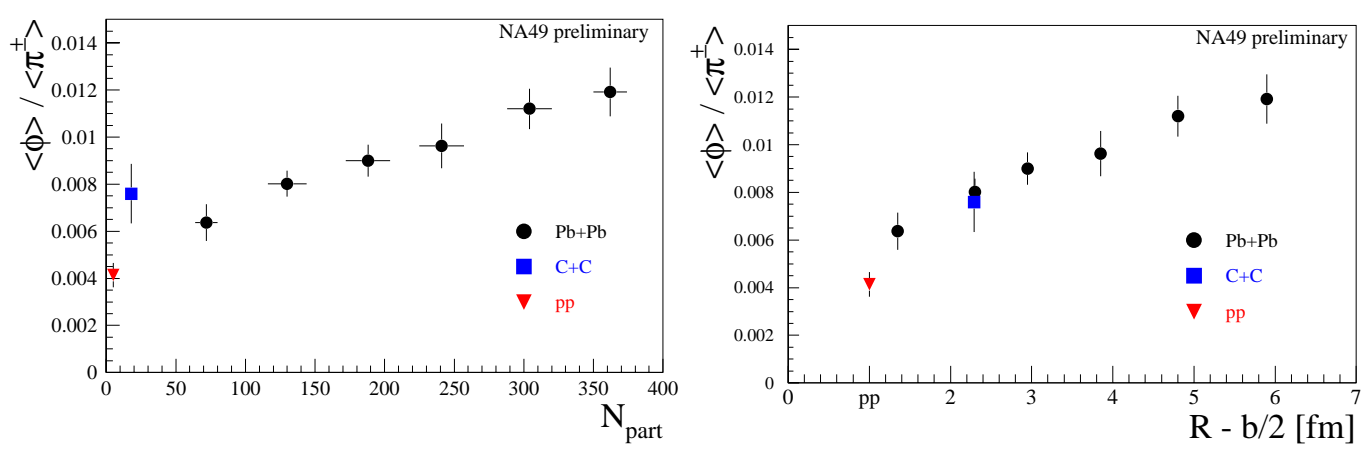

Figure 5: The dependence of $\langle\phi\rangle /\left\langle\pi^{ \pm}\right\rangle$on the system size (using $\mathrm{p}+\mathrm{p}, \mathrm{C}+\mathrm{C}$, and $\mathrm{Pb}+\mathrm{Pb}$ ). In the left panel the dependence on the number of participants is shown. The right panel shows the dependence on $R-b / 2$ (see text).

New results on $\phi$ production in central $\mathrm{C}+\mathrm{C}$ collisions and $\mathrm{Pb}+\mathrm{Pb}$ collisions at different centrality at $158 \mathrm{~A} \cdot \mathrm{GeV}$ are presented in Fig. $\frac{1}{2}\left(\left\langle\pi^{+}\right\rangle+\left\langle\pi^{-}\right\rangle\right)$. The $\mathrm{p}+\mathrm{p}$ result is taken from [1] $\left.{ }_{1}^{2} \overline{1}\right]$, where further details of the analysis can also be found. It is seen that $\langle\phi\rangle /\left\langle\pi^{ \pm}\right\rangle$in $\mathrm{C}+\mathrm{C}$ collisions is closer to the value for central $\mathrm{Pb}+\mathrm{Pb}$ than one would expect from the number of participants. This could be related to the geometry of the interaction zone. If we denote $R$ the radius of the nucleus and $b$ the impact parameter of the collision, the thickness of the interaction region is given by $R-b / 2$. Plotting the data as a function of this measure of centrality, (see right panel of Fig. 留), the $\mathrm{C}+\mathrm{C}$ data follow the trend of the $\mathrm{Pb}+\mathrm{Pb}$ data. Note that the surface to volume ratio is closely related to the thickness of the interaction region [i] $\overline{1} \overline{3}]$.

\section{Summary}

The data on kaon production in central $\mathrm{Pb}+\mathrm{Pb}$ collisions show a maximum in the $\left\langle K^{+}\right\rangle /\left\langle\pi^{+}\right\rangle$ ratio around $40 \mathrm{~A} \cdot \mathrm{GeV}$. The closely related strangeness to entropy ratio will also have a maximum at this energy, which might be related to the phase transition to the QGP [i.

The $\Xi$ and $\Lambda$ (anti-)hyperon yields at mid-rapidity in $\mathrm{p}+\mathrm{A}$ collisions are larger than one would expect assuming wounded nucleon scaling starting from minimum bias $\mathrm{p}+\mathrm{p}$ collisions. 
The system size dependence for the $\phi$ yield clearly shows that $\phi$ production does not scale with the number of participants.

\section{Acknowledgments}

This work was supported by the Director, Office of Energy Research, Division of Nuclear Physics of the Office of High Energy and Nuclear Physics of the US Department of Energy (DE-ACO3-76SFOOO98 and DE-FG02-91ER40609), the US National Science Foundation, the Bundesministerium fur Bildung und Forschung, Germany, the Alexander von Humboldt Foundation, the UK Engineering and Physical Sciences Research Council, the Polish State Committee for Scientific Research (5 P03B 13820 and 2 P03B 02418), the Hungarian Scientific Research Foundation (T14920 and T23790), the EC Marie Curie Foundation, and the Polish-German Foundation.

\section{References}

[1] MILC Collaboration, C. W. Bernard et. al., QCD thermodynamics with an improved lattice action, Phys. Rev. D56 (1997) 5584-5595, [hep-lat/9703003].

[2] K. Rajagopal, Mapping the QCD phase diagram, Nucl. Phys. A661 (1999) 150-161, $[$ [hep-ph/9908360i].

[3] NA49 Collaboration, S. Margetis et. al., Transverse energy production in $\mathrm{Pb}-208+\mathrm{Pb}$ collisions at 158-GeV per nucleon, Phys. Rev. Lett. 75 (1995) 3814-3817.

[4] J. Rafelski, Formation and observables of the quark - gluon plasma, Phys. Rept. 88 (1982) 331.

[5] NA49 Collaboration, S. Afanasev et. al., The NA49 large acceptance hadron detector, Nucl. Instrum. Meth. A430 (1999) 210-244.

[6] M. K. Hegab and J. Hufner, The distribution of gray particles in very high-energy hadron nucleus collisions, Nucl. Phys. A384 (1982) 353.

[7] M. Gazdzicki and M. I. Gorenstein, On the early stage of nucleus nucleus collisions, Acta Phys. Polon. B30 (1999) 2705, [hep-ph/9803462].

[8] A. Wroblewski, On the strange quark suppression factor in high-energy collisions, Acta Phys. Polon. B16 (1985) 379-392.

[9] WA97 Collaboration, E. Andersen et. al., Strangeness enhancement at mid-rapidity in $\mathrm{Pb} \mathrm{Pb}$ collisions at 158-A-GeV/c, Phys. Lett. B449 (1999) 401-406.

[10] NA49 Collaboration, S. V. Afanasev et. al., Production of multi-strange hyperons and strange resonances in the NA49 experiment, J. Phys. G27 (2001) 367-374.

[11] NA49 Collaboration, T. Susa, Cascade baryon production in $p+p, p+A$ and $A+A$ interactions at $158 \mathrm{AGeV}$, to appear in proceedings of Quark Matter 2001, Brookhaven.

[12] NA49 Collaboration, S. V. Afanasev et. al., Production of $\phi$-mesons in $p+p, p+P b$ and central $P b+P b$ collisions at $E($ beam $)=158-A-G e V$, Phys. Lett. B491 (2000) 59-66.

[13] NA49 Collaboration, F. Sikler, Recent NA49 results on Pb $+P b$ collisions at CERN SPS, hep-ex/0102004. Proceedings ISMD2000 Tihany. 